\title{
Partition-theoretic interpretations of some $q$-series identities of Ramanujan
}

\author{
Nipen Saikia \\ Dept. of Mathematics, Rajiv Gandhi University, \\ Rono Hills, Doimukh-791112, Arunachal Pradesh, India. \\ Corresponding author: nipennak@yahoo.com
}

\begin{abstract}
Ramanujan's lost notebook contains several $q$-series identities, and some of them have theta-function representations. We give partition-theoretic interpretations of some of these identities and prove Ramanujan-type congruences for certain partition functions.
\end{abstract}

Keywords: Congruence; Jacobi's triple product identity; partition of integer; $q$-series; Ramanujan's theta-functions.

\section{Introduction}

Ramanujan's lost notebook contains several $q$-series identities. Details of these can be found in (Andrews \& Berndt, 2009). The purpose of this paper is to give partition interpretations of some of the $q$-series identities and prove Ramanujan-type congruences for certain par-tition functions.

In the sequel, throughout the paper, we assume that $|q|<1$ always and as usual, for any complex number $a$ and $q$, we set

$(a ; q)_{0}=1, \quad(a ; q)_{n}=\prod_{k=0}^{n-1}\left(1-a q^{k}\right)$, for $n \geq 1$,

and

$$
(a ; q)_{\infty}=\prod_{k=0}^{\infty}\left(1-a q^{k}\right) .
$$

For brevity, we write

$$
\begin{array}{r}
\left(a_{1} ; q\right)_{\infty}\left(a_{2} ; q\right)_{\infty}\left(a_{3} ; q\right)_{\infty} \cdots\left(a_{m} ; q\right)_{\infty} \\
=\left(a_{1}, a_{2}, a_{3}, \cdots, a_{m} ; q\right)_{\dot{\infty}}
\end{array}
$$

Several $q$-series identities in Ramanujanpost notebook have representations in terms of Ramanujan's theta-function $\quad f(a, b)$ (Berndt ,1991). p. 34, (18.1)) defined by

$f(a, b)=\sum_{k=-\infty}^{\infty} a^{k(k+1) / 2} b^{k(k-1) / 2}, \quad|a b|<1$.

In terms of $f(a, b)$, Jacobi's triple product identity (Berndt, 1991, p. 35, Entry 19) can be stated as

$f(a, b)=(-a ; a b)_{\infty}(-b ; a b)_{\infty}(a b ; a b)_{\infty}$

$$
=(-a,-b, a b ; a b)_{\infty} .
$$

Three important special cases of $f(a, b)$ are the theta-functions $\phi(q), \psi(q)$ and $f(-q)$ (Berndt, 1991, p. 36, Entry 22), which are given by

$\phi(q):=f(q, q)=\sum_{n=-\infty}^{\infty} q^{n^{2}}=\frac{\left(q^{2} ; q^{2}\right)_{\infty}^{5}}{(q ; q)_{\infty}^{2}\left(q^{4} ; q^{4}\right)_{\infty}^{2}}$,

$\psi(q):=f\left(q, q^{3}\right)=\sum_{k=0}^{\infty} q^{k(k+1) / 2}=\frac{\left(q^{2} ; q^{2}\right)_{\infty}^{2}}{(q ; q)_{\infty}}$

and

$$
\begin{aligned}
f(-q):=f(-q, & \left.-q^{2}\right) \sum_{n=-\infty}^{\infty}(-1)^{n} q^{n(3 n-1) / 2} \\
& =(q ; q)_{\infty}
\end{aligned}
$$


respectively. Ramanujan also defined the function $\chi(q)$ (Berndt, 1991, p. 39, Entry 22(iv)) as

$$
\chi(q)=\left(-q ; q^{2}\right)_{\infty}=\frac{\left(q^{2} ; q^{2}\right)_{\infty}^{2}}{(q ; q)_{\infty}\left(q^{4} ; q^{4}\right)_{\infty}} .
$$

By using elementary $q$-analysis, one can easily verify that

$\phi(-q)=\frac{(q ; q)_{\infty}^{2}}{\left(q^{2} ; q^{2}\right)_{\infty}}, \psi(-q)=\frac{(q ; q)_{\infty}\left(q^{4} ; q^{4}\right)_{\infty}}{\left(q^{2} ; q^{2}\right)_{\infty}}$ and

$$
\chi(-q)=\frac{(q ; q)_{\infty}^{2}}{\left(q^{2} ; q^{2}\right)_{\infty}}
$$

With this background, we now state some $q$ series identities from (Andrews \& Berndt, 2009) for which we will give partitiontheoretic interpretations in this paper.

(i) (Andrews \& Berndt, 2009, p. 32, Entry 1.7.5):

$$
\sum_{n=0}^{\infty} \frac{\left(-q^{2} ; q^{2}\right)_{n} q^{n(n+1) / 2}}{(q ; q)_{n}\left(q ; q^{2}\right)_{n}}=\frac{\psi\left(-q^{2}\right)}{\phi(-q)} .
$$

(ii) (Andrews \& Berndt, 2009, p. 32, Entry 1.7.6):

$$
\sum_{n=0}^{\infty} \frac{\left(-q ; q^{2}\right)_{n} q^{\left(n^{2}+3 n\right) / 2}}{(q ; q)_{n}\left(q ; q^{2}\right)_{n+1}}=\frac{f\left(-q,-q^{7}\right)}{\phi(-q)}
$$

(iii) (Andrews \& Berndt, 2009, p. 34, Entry 1.7.8):

$$
\sum_{n=0}^{\infty} \frac{\left(-q ; q^{2}\right)_{n} q^{n(n+1) / 2}}{(q ; q)_{n}\left(q ; q^{2}\right)_{n+1}}=\frac{f\left(-q^{3},-q^{5}\right)}{\phi(-q)}
$$

(iv) (Andrews \& Berndt, 2009, p. 48, Entry 2.3.3):

$$
\sum_{n=0}^{\infty} \frac{\left(-q ; q^{2}\right)_{n}^{2} q^{n(n+1) / 2}}{(q ; q)_{n}\left(q ; q^{2}\right)_{n+1}}=\frac{\psi\left(q^{2}\right)}{\phi(-q)} .
$$

(v) (Andrews \& Berndt, 2009, p. 65, Entry 3.4.5):

$$
\sum_{n=0}^{\infty} \frac{\left(-q ; q^{2}\right)_{n} q^{n}}{(q ; q)_{2 n+1}}=\frac{\psi\left(-q^{3}\right)}{\phi(-q)}
$$

(vi) (Andrews \& Berndt, 2009, p. 85, Entry 4.2.8):

$$
\sum_{n=0}^{\infty} \frac{(-1 ; q)_{n} q^{n^{2}}}{(q ; q)_{n}\left(q ; q^{2}\right)_{n}}=\frac{\phi\left(-q^{3}\right)}{\phi(-q)}
$$

(vii) (Andrews \& Berndt, 2009, p. 87,

Entry 4.2.12):

$$
\sum_{n=0}^{\infty} \frac{(-q ; q)_{n} q^{n^{2}+n}}{(q ; q)_{n}\left(q ; q^{2}\right)_{n+1}}=\frac{f\left(-q,-q^{5}\right)}{\phi(-q)} .
$$

(viii) (Andrews \& Berndt, 2009, p. 88, En-try 4.2.13):

$$
\sum_{n=0}^{\infty} \frac{\left(-q^{2} ; q^{2}\right)_{n} q^{n(n+1)}}{\left(q ; q^{2}\right)_{2 n+1}}=\frac{f\left(q, q^{5}\right)}{\phi\left(-q^{2}\right)} .
$$

We end this section by defining partition of a positive integer. A partition of a positive integer $n$ is a non-increasing sequence of positive integers, called parts, whose sum equals $n$. For example, $n$ $=3$ has three partitions, namely,

$$
3, \quad 2+1, \quad 1+1+1 \text {. }
$$

If $p(n)$ denote the number of partitions of $n$, then $p(3)=3$. The generating function for $p(n)$ due to Euler is given by

$$
\sum_{n=0}^{\infty} p(n) q^{n}=\frac{1}{(q ; q)_{\infty}} .
$$

Ramanujan (Ramanujan, 1919) established fol-lowing beautiful congruences for $p(n)$ :

$$
\begin{gathered}
p(5 n+4) \equiv 0(\bmod 5), \\
p(7 n+5) \equiv 0 \quad(\bmod 7)
\end{gathered}
$$

and

$$
p(11 n+6) \equiv 0 \quad(\bmod 11) .
$$

A part in a partition of $n$ has $r$ colours if there are $r$ copies of each part available and all of them are viewed as distinct objects. For any positive integers $n$ and $r$, let $p_{r}(n)$ denote the number of partitions of $n$ where each part may have $r$ distinct colours. For example, if each part in the partition of 3 has two colours, say red (indicated by the suffix $r$ ) and green (indicated by the suffix $g$ ), then the number of two colour partitions of 3 is 10 , namely

$$
\begin{gathered}
3_{r}, \quad 3_{g}, \quad 2_{r}+1_{r}, \quad 2_{r}+1_{g}, \quad 2_{g}+1_{g}, \\
2_{g}+1_{r}, \quad 1_{r}+1_{r}+1_{r}, \quad 1_{g}+1_{g}+1_{g}, \\
1_{r}+1_{g}+1_{g}, \quad 1_{r}+1_{r}+1_{g} .
\end{gathered}
$$

The generating function of $p_{r}(n)$ (Berndt \& Rankin, 1995) is given by

$$
\sum_{n=0}^{\infty} p_{r}(n) q^{n}=\frac{1}{(q ; q)_{\infty}^{r}} .
$$

For $r=1, p_{1}(n)$ is the usual unrestricted partition function $p(n)$ defined in (18).

We also note that, for positive integers $k, m$ and $r$,

$$
\frac{1}{\left(q^{k} ; q^{m}\right)_{\infty}^{r}}
$$


is the generating function of the number of partitions of a positive integer with parts congruent to $k$ modulo $m$ (that is, $\equiv k(\bmod$ $m)$ ) and each part has $r$ colours. Similarly,

$$
\frac{1}{\left(q^{k_{1}} ; q^{m}\right)_{\infty}^{2}\left(q^{k_{2}} ; q^{m}\right)_{\infty}^{2}}=\frac{1}{\left(q^{k_{1}}, q^{k_{2}} ; q^{m}\right)_{\infty}^{2}}
$$

is the generating function of the number of partitions of positive integer with parts $\equiv k_{1}$ or $k_{2}$ $(\bmod m)$ and each part has two colours.

\section{Partition-Theoretic Interpretations of $q$ - Series Identities}

In this section, we call $q^{m}$ as the base of the $q$-product $\left(q^{k} ; q^{m}\right)_{\infty}$ for positive integer $m$.

Theorem 2.1. Let $A_{1}(n)$ denote the number of partitions of a positive integer $n$ where the parts are $\equiv 1,3,4,5$ or $7(\bmod 8)$ and each part has two colours except the parts $\equiv 4$ $(\bmod 8)$. Then

$$
\begin{aligned}
\sum_{n=0}^{\infty} A_{1}(n) q^{n} & =\sum_{n=0}^{\infty} \frac{\left(-q^{2} ; q^{2}\right)_{n} q^{n(n+1) / 2}}{(q ; q)_{n}\left(q ; q^{2}\right)_{n}} \\
& =\frac{\psi\left(-q^{2}\right)}{\phi(-q)}
\end{aligned}
$$

Proof. From (9), we see that

$$
\frac{\psi\left(-q^{2}\right)}{\phi(-q)}=\frac{\left(q^{2} ; q^{2}\right)_{\infty}^{2}\left(q^{8} ; q^{8}\right)_{\infty}}{\left(q^{4} ; q^{4}\right)_{\infty}(q ; q)_{\infty}^{2}}
$$

Changing the base $q$ in $(q ; q)_{\infty}$ to $q^{8}$, we obtain

$$
\begin{array}{r}
\left(q^{2} ; q^{2}\right)_{\infty}=\left(q^{2} ; q^{8}\right)_{\infty}\left(q^{4} ; q^{8}\right)_{\infty}\left(q^{6} ; q^{8}\right)_{\infty}\left(q^{8} ; q^{8}\right)_{\infty} \\
=\left(q^{2}, q^{4}, q^{6}, q^{8} ; q^{8}\right)_{\infty} .(23)
\end{array}
$$

Similarly, by changing the bases to $q^{8}$ in $\left(q^{4} ; q^{4}\right)_{\infty}$ and $(q ; q)_{\infty}$, we obtain

$$
\left(q^{4} ; q^{4}\right)_{\infty}=\left(q^{4}, q^{8} ; q^{8}\right)_{\infty}
$$

and

$$
(q ; q)_{\infty}=\left(q, q^{2}, q^{3}, q^{4}, q^{5}, q^{6}, q^{7}, q^{8} ; q^{8}\right)_{\infty} .
$$

Employing (23), (24) and (25) in (22) and simplifying, we arrive at

$$
\frac{\psi\left(-q^{2}\right)}{\phi(-q)}=\frac{1}{\left(q^{4} ; q^{8}\right)_{\infty}\left(q, q^{3}, q^{5}, q^{7} ; q^{8}\right)_{\infty}^{2}} .
$$

Now the desired result follows easily from (10) and (26).
Theorem 2.2. Let $A_{2}(n)$ denote the number of partitions of a positive integer $n$ with parts $\not \equiv 0$ $(\bmod 8)$ and parts $\equiv 3$ or $5(\bmod 8)$ have two colours. Then

$$
\begin{aligned}
\sum_{n=0}^{\infty} A_{2}(n) q^{n} & =\sum_{n=0}^{\infty} \frac{\left(-q ; q^{2}\right)_{n} q^{\left(n^{2}+3 n\right) / 2}}{(q ; q)_{n}\left(q ; q^{2}\right)_{n+1}} \\
& =\frac{f\left(-q,-q^{7}\right)}{\phi(-q)} .
\end{aligned}
$$

Proof. Using (4) and (9), we see that

$$
\frac{f\left(-q,-q^{7}\right)}{\phi(-q)}=\frac{\left(q, q^{7}, q^{8} ; q^{8}\right)_{\infty}\left(q^{2} ; q^{2}\right)_{\infty}}{(q ; q)_{\infty}^{2}} .
$$

Changing the bases of $q$-products to $q^{8}$, we find that

$$
\begin{aligned}
& \frac{f\left(-q,-q^{7}\right)}{\phi(-q)} \\
= & \frac{\left(q, q^{7}, q^{8} ; q^{8}\right)_{\infty}\left(q^{2}, q^{4}, q^{6}, q^{8} ; q^{8}\right)_{\infty}}{\left(q, q^{2}, q^{3}, q^{4}, q^{5}, q^{6}, q^{7}, q^{8} ; q^{8}\right)_{\infty}^{2}} .
\end{aligned}
$$

Simplifying (28), we obtain

$$
\begin{aligned}
& \frac{f\left(-q,-q^{7}\right)}{\phi(-q)} \\
= & \frac{1}{\left(q, q^{2}, q^{4}, q^{6}, q^{7} ; q^{8}\right)_{\infty}\left(q^{3}, q^{5} ; q^{8}\right)_{\infty}^{2}}
\end{aligned}
$$

Now the desired result follows easily from (11) and (29).

Theorem 2.3. Let $A_{3}(n)$ denote the number of partitions of a positive integer $n$ with parts $\not \equiv 0$ $(\bmod 8)$ and parts $\equiv 1$ or $7(\bmod 8)$ have two colours. Then

$$
\begin{aligned}
\sum_{n=0}^{\infty} A_{3}(n) q^{n} & =\sum_{n=0}^{\infty} \frac{\left(-q ; q^{2}\right)_{n} q^{n(n+1) / 2}}{(q ; q)_{n}\left(q ; q^{2}\right)_{n+1}} \\
& =\frac{f\left(-q^{3},-q^{5}\right)}{\phi(-q)}
\end{aligned}
$$

Proof. Using (4) and (9), we see that

$\frac{f\left(-q^{3},-q^{5}\right)}{\phi(-q)}=\frac{\left(q^{3}, q^{5}, q^{8} ; q^{8}\right)_{\infty}\left(q^{2} ; q^{2}\right)_{\infty}}{(q ; q)_{\infty}^{2}}$.

Changing the bases of $q$-products to $q^{8}$, we find that

$$
\frac{f\left(-q^{3},-q^{5}\right)}{\phi(-q)}
$$

$$
=\frac{\left(q^{3}, q^{5}, q^{8} ; q^{8}\right)_{\infty}\left(q^{2}, q^{4}, q^{6}, q^{8} ; q^{8}\right)_{\infty}}{\left(q, q^{2}, q^{3}, q^{4}, q^{5}, q^{6}, q^{7}, q^{8} ; q^{8}\right)_{\infty}^{2}} .
$$


Simplifying (31), we obtain

$$
\begin{aligned}
& \frac{f\left(-q^{3},-q^{5}\right)}{\phi(-q)} \\
= & \frac{1}{\left(q^{2}, q^{3}, q^{4}, q^{5}, q^{6} ; q^{8}\right)_{\infty}\left(q, q^{7} ; q^{8}\right)_{\infty}^{2}} .
\end{aligned}
$$

Now the desired result follows easily from (12) and (32).

Theorem 2.4. Let $A_{4}(n)$ denote the number of partitions of a positive integer $n$ with parts $\not \equiv 0$ $(\bmod 4)$ and each part has two colours. Then

$$
\begin{aligned}
\sum_{n=0}^{\infty} A_{4}(n) q^{n} & =\sum_{n=0}^{\infty} \frac{\left(-q ; q^{2}\right)_{n}^{2} q^{n(n+1) / 2}}{(q ; q)_{n}\left(q ; q^{2}\right)_{n+1}} \\
& =\frac{\psi\left(q^{2}\right)}{\phi(-q)} .
\end{aligned}
$$

Proof. Using (6) and (9), we see that

$$
\frac{\psi\left(q^{2}\right)}{\phi(-q)}=\frac{\left(q^{4} ; q^{4}\right)_{\infty}^{2}}{(q ; q)_{\infty}^{2}}
$$

Changing the base $q$ in $(q ; q)_{\infty}$ to $q^{4}$ of (33) and simplifying, we obtain

$$
\frac{\psi\left(q^{2}\right)}{\phi(-q)}=\frac{1}{\left(q, q^{2}, q^{3} ; q^{4}\right)_{\infty}^{2}}
$$

Now the desired result follows from (13) and (34).

Theorem 2.5. Let $A_{5}(n)$ denote the number of partitions of a positive integer $n$ with parts $\not \equiv 0$ $(\bmod 12)$ and parts $\equiv 1,5,7$ or $11(\bmod 12)$ have two colours. Then

$$
\sum_{n=0}^{\infty} A_{5}(n) q^{n}=\sum_{n=0}^{\infty} \frac{\left(-q ; q^{2}\right)_{n} q^{n}}{(q ; q)_{2 n+1}}=\frac{\psi\left(-q^{3}\right)}{\phi(-q)} .
$$

Proof. Using (9), we see that

$$
\frac{\psi\left(-q^{3}\right)}{\phi(-q)}=\frac{\left(q^{3} ; q^{3}\right)_{\infty}\left(q^{12} ; q^{12}\right)_{\infty}\left(q^{2} ; q^{2}\right)_{\infty}}{\left(q^{4} ; q^{4}\right)_{\infty}(q ; q)_{\infty}^{2}} .
$$

Changing the bases to $q^{12}$ in (35) and simplifying, we obtain

$$
\begin{aligned}
\frac{\psi\left(-q^{3}\right)}{\phi(-q)} & =\frac{1}{\left(q^{2}, q^{3}, q^{4}, q^{6}, q^{8}, q^{9}, q^{10} ; q^{12}\right)_{\infty}} \\
& \times \frac{1}{\left(q, q^{5}, q^{7}, q^{11} ; q^{12}\right)_{\infty}^{2}} .
\end{aligned}
$$

Now the desired result follows from (14) and (36).

Theorem 2.6. Let $A_{6}(n)$ denote the number of partitions of a positive integer $n$ with parts $\equiv 1,2,4$ or $5(\bmod 6)$ and parts $\equiv 1$ or 5

(mod 6) have two colours. Then

$\sum_{n=0}^{\infty} A_{6}(n) q^{n}=\sum_{n=0}^{\infty} \frac{(-1 ; q)_{n} q^{n^{2}}}{(q ; q)_{n}\left(q ; q^{2}\right)_{n}}=\frac{\phi\left(-q^{3}\right)}{\phi(-q)}$.

Proof. Using (9), we find that

$$
\frac{\phi\left(-q^{3}\right)}{\phi(-q)}=\frac{\left(q^{3} ; q^{3}\right)_{\infty}^{2}\left(q^{2} ; q_{\infty}^{2}\right)}{\left(q^{6} ; q^{6}\right)_{\infty}(q ; q)_{\infty}^{2}}
$$

Changing the bases ${ }^{q}, q$ and $q$ in (37) to $q^{6}$ and simplifying, we obtain

$$
\frac{\phi\left(-q^{3}\right)}{\phi(-q)}=\frac{1}{\left(q^{2}, q^{4} ; q^{6}\right)_{\infty}\left(q, q^{5} ; q^{6}\right)_{\infty}^{2}}
$$

Now the desired result follows from (15) and (38).

Theorem 2.7. Let $A_{7}(n)$ denote the number of partitions of a positive integer $n$ with parts $\not \equiv$ $0(\bmod 6)$ and parts $\equiv 3(\bmod 6)$ have two colours. Then

$$
\begin{aligned}
\sum_{n=0}^{\infty} A_{7}(n) q^{n} & =\sum_{n=0}^{\infty} \frac{(-q ; q)_{n} q^{n^{2}+n}}{(q ; q)_{n}\left(q ; q^{2}\right)_{n+1}} \\
& =\frac{f\left(-q,-q^{5}\right)}{\phi(-q)} .
\end{aligned}
$$

Proof. Using (4) and (9), we see that

$$
\frac{f\left(-q,-q^{5}\right)}{\phi(-q)}=\frac{\left(q, q^{5}, q^{6} ; q^{6}\right)_{\infty}\left(q^{2} ; q^{2}\right)_{\infty}}{(q ; q)_{\infty}^{2}}
$$

Changing the bases of $q$-products to $q^{6}$ in (39), we obtain

$$
\begin{aligned}
& \frac{f\left(-q,-q^{5}\right)}{\phi(-q)} \\
= & \frac{\left(q, q^{5}, q^{6} ; q^{6}\right)_{\infty}\left(q^{2}, q^{4}, q^{6} ; q^{6}\right)_{\infty}}{\left(q, q^{2}, q^{3}, q^{4}, q^{5}, q^{6} ; q^{6}\right)_{\infty}^{2}} .
\end{aligned}
$$

Simplifying (40), we obtain

$\frac{f\left(-q,-q^{5}\right)}{\phi(-q)}=\frac{1}{\left(q, q^{2}, q^{4}, q^{5} ; q^{6}\right)_{\infty}\left(q^{3} ; q^{6}\right)_{\infty}^{2}}$.

Now the desired result follows easily from (16) and (41).

Theorem 2.8. Let $A_{8}(n)$ denote the number of partitions of a positive integer $n$ with parts $\not \equiv$ 0,3,9 $(\bmod 12)$. Then

$$
\sum_{n=0}^{\infty} \frac{\left(-q^{2} ; q^{2}\right)_{n} q^{n(n+1)}}{\left(q ; q^{2}\right)_{2 n+1}}=\frac{f\left(q, q^{5}\right)}{\phi\left(-q^{2}\right)} .
$$


Proof. From (Berndt, 1991, p. 51,

Example(v)), we note that

$$
f\left(q, q^{5}\right)=\psi\left(-q^{3}\right) \chi(q)
$$

Using (8), (9) and (42), we obtain

$$
\begin{aligned}
\frac{f\left(q, q^{5}\right)}{\phi\left(-q^{2}\right)} & =\frac{\psi\left(-q^{3}\right) \chi(q)}{\phi\left(-q^{2}\right)} \\
& =\frac{\left(q^{3}, q^{3}\right)_{\infty}\left(q^{12} ; q_{\infty}^{12}\right.}{(q ; q)_{\infty}\left(q^{6} ; q^{6}\right)_{\infty}} .
\end{aligned}
$$

Changing the bases of $q$-products to $q^{12}$ in (43) and simplifying, we obtain

$$
\begin{aligned}
& \frac{\frac{f\left(q, q^{5}\right)}{\phi\left(-q^{2}\right)}}{=} \frac{1}{\left(q, q^{2}, q^{4}, q^{5}, q^{6}, q^{7}, q^{8}, q^{10}, q^{11} ; q^{12}\right)_{\infty}} .
\end{aligned}
$$

The desired result follows easily from (17) and (44).

\section{Ramanujan-Type Congruences for $A_{1}(n), A_{4}(n), A_{5}(n)$ and $A_{6}(n)$}

In this section, the suffixes ' $r$ ' and ' $g$ ' in parts of partitions will indicate two colours red and green of the parts of the partitions, respectively.

Theorem 31. If $A_{1}(n)$ is as defined in Theorem 2.1, then

$$
A_{1}(2 n+1) \equiv 0(\bmod 2) \text {. }
$$

Proof. From (Hirschhorn \& Sellers, 2005), we note that

$$
\begin{aligned}
& \frac{1}{\phi(-q)}=\frac{1}{\phi\left(q^{4}\right)^{4}}\left(\phi\left(q^{4}\right)^{3}+2 q \phi\left(q^{4}\right)^{2} \psi\left(q^{8}\right)\right. \\
& \left.\quad+4 q^{2} \phi\left(q^{4}\right) \psi\left(q^{8}\right)^{2}+8 q^{3} \psi\left(q^{8}\right)^{3}\right)
\end{aligned}
$$

Employing (45) in Theorem 2.1, we obtain

$$
\begin{gathered}
\sum_{n=0}^{\infty} A_{1}(n) q^{n}=\frac{\psi\left(-q^{2}\right)}{\phi\left(q^{4}\right)^{4}}\left(\phi\left(q^{4}\right)^{3}+2 q \phi\left(q^{4}\right)^{2} \psi\left(q^{8}\right)\right. \\
\left.+4 q^{2} \phi\left(q^{4}\right) \psi\left(q^{8}\right)^{2}+8 q^{3} \psi\left(q^{8}\right)^{3}\right) \\
\equiv \frac{\psi\left(-q^{2}\right)}{\phi\left(q^{4}\right)} \quad(\bmod 2)
\end{gathered}
$$

Since right hand side of (46) contains no terms involving $q^{2 n+1}$, extracting the terms involving $q^{2 n+1}$ from (46), we arrive at the desired result.
Remark 3.2. We note that $A_{1}(3)=6$ with the relevant partitions given by $3_{r}, 3_{g}, 1_{r}+1_{r}+$ $1_{r}, 1_{r}+1_{r}+1_{g}, 1_{r}+1_{g}+1_{g}$ and $1_{g}+1_{g}+1_{g}$. This verifies Theorem 3.1.

Theorem 33. If $A_{4}(n)$ is as defined in Theorem 2.4, then

$$
A_{4}(2 n+1) \equiv 0(\bmod 2) .
$$

Proof. Employing (45) in Theorem 2.4, we obtain

$$
\begin{gathered}
\sum_{n=0}^{\infty} A_{4}(n) q^{n}=\frac{\psi\left(q^{2}\right)}{\phi\left(q^{4}\right)^{4}}\left(\phi\left(q^{4}\right)^{3}+2 q \phi\left(q^{4}\right)^{2} \psi\left(q^{8}\right)\right. \\
\left.+4 q^{2} \phi\left(q^{4}\right) \psi\left(q^{8}\right)^{2}+8 q^{3} \psi\left(q^{8}\right)^{3}\right) \\
\equiv \frac{\psi\left(q^{2}\right)}{\phi\left(q^{4}\right)} \quad(\bmod 2)
\end{gathered}
$$

Extracting the terms involving $q^{2 n+1}$ from (47), we arrive at the desired result.

Remark 3.4. We note that $A_{4}(3)=10$ and the relevant partitions are $3_{r}, 3_{g}, 2_{r}+1_{r}, 2_{g}+1_{g}$, $2_{r}+1_{g}, 2_{g}+1_{r}, 1_{r}+1_{r}+1_{r}, 1_{r}+1_{r}+1_{g}, 1_{r}$ $+1_{g}+1_{g}$ and $1_{g}+1_{g}+1_{g}$. This verifies Theorem 3.3.

Theorem 35. If $A_{5}(n)$ is as defined in Theorem 2.5 , then

$$
\begin{aligned}
& \text { (i) } A_{5}(3 n+1) \equiv 0 \quad(\bmod 2), \\
& \text { (ii) } A_{5}(3 n+2) \equiv 0 \quad(\bmod 4) .
\end{aligned}
$$

Proof. From (Hirschhorn \& Sellers, 2005), we note that

$$
\begin{gathered}
\frac{1}{\phi(-q)}=\frac{\phi\left(-q^{9}\right)}{\phi\left(-q^{3}\right)^{4}}\left(\phi\left(-q^{9}\right)^{2}\right. \\
\left.+2 q \phi\left(-q^{9}\right) \omega\left(-q^{3}\right)+4 q^{2} \omega\left(-q^{3}\right)^{2}\right), \\
\text { where } \omega(-q)=\frac{(q ; q)_{\infty}\left(q^{6} ; q^{6}\right)_{\infty}^{2}}{\left(q^{2} ; q^{2}\right)_{\infty}\left(q^{3} ; q^{3}\right)_{\infty}} .
\end{gathered}
$$

Employing (48) in Theorem 2.5, we obtain

$$
\begin{aligned}
& \sum_{n=0}^{\infty} A_{5}(n) q^{n}=\frac{\psi\left(-q^{3}\right) \phi\left(-q^{9}\right)}{\phi\left(-q^{3}\right)^{4}}\left(\phi\left(-q^{9}\right)^{2}\right. \\
& \left.+2 q \phi\left(-q^{9}\right) \omega\left(-q^{3}\right)+4 q^{2} \omega\left(-q^{3}\right)^{2}\right) .
\end{aligned}
$$

Extracting the terms involving $q^{3 n+1}$ and $q^{3 n+2}$ from (49), we arrive at (i) and (ii), respectively. 
Remark 3.6. We note that, $A_{5}(2)=4$ with relevant partitions given by $2,1_{r}+1_{r}, 1_{r}+1_{g}$ and $1_{g}+1_{g}$. This verifies Theorem 3.5(ii) for $n=0$.

Theorem 3.7. If $A_{6}(n)$ is as defined in Theorem 2.6, then

$$
\begin{aligned}
\text { (i) } A_{6}(3 n+1) & \equiv 0 \quad(\bmod 2), \\
\left(\text { ii } A_{6}(3 n+2)\right. & \equiv 0 \quad(\bmod 4), \\
\text { (iii) } A_{6}(9 n+3 j) & \equiv 0 \quad(\bmod 3), \\
\text { where } j & =1,2,
\end{aligned}
$$$$
\text { (iv) } A_{6}(9 n) \equiv A_{6}(n) \quad(\bmod 3) \text {. }
$$

Proof. Employing (48) in Theorem 2.6, we obtain

$$
\begin{gathered}
\sum_{n=0}^{\infty} A_{6}(n) q^{n}=\frac{\phi\left(-q^{9}\right)}{\phi\left(-q^{3}\right)^{3}}\left(\phi\left(-q^{9}\right)^{2}\right. \\
\left.+2 q \phi\left(-q^{9}\right) \omega\left(-q^{3}\right)+4 q^{2} \omega\left(-q^{3}\right)^{2}\right) .
\end{gathered}
$$

Extracting the terms involving $q^{3 n+1}$ and $q^{3 n+2}$ from (50), we arrive at (i) and (ii), respectively.

Next, extracting the terms involving $q^{3 n}$ from (50) and replacing $q^{3}$ by $q$, we obtain

$\sum_{n=0}^{\infty} A_{6}(3 n) q^{n}=\frac{\phi\left(-q^{3}\right)^{3}}{\phi(-q)^{3}} \equiv \frac{\phi\left(-q^{9}\right)}{\phi\left(-q^{3}\right)}(\bmod 3),(51)$

where we used the result

$$
\phi\left(-q^{3}\right) \equiv \phi(-q)^{3} \quad(\bmod 3)
$$

which follows from (1), (9), and the binomial theorem.

Since right hand side of (51) contains no terms involving $q^{3 n+1}$ and $q^{3 n+2}$, so extracting the terms involving $q^{3 n+j}$ for $j=1$, 2 from (51), we arrive at (iii).

To prove (iv), extracting the terms involving $q^{3 n}$ from (51), replacing $q^{3}$ by $q$ and employing Theorem 2.6, we obtain

$$
\begin{aligned}
& \sum_{n=0}^{\infty} A_{6}(9 n) q^{n} \equiv \frac{\phi\left(-q^{3}\right)}{\phi(-q)} \\
&=\sum_{n=0}^{\infty} A_{6}(n) q^{n} \quad(\bmod 3) .
\end{aligned}
$$

Extracting the coefficient of $q^{n}$ on both sides of (53), we arrive at (iv).
Remark 3.8. We note that, $A_{6}(3)=6$ and the relevant partitions are given by $2+1_{r}, \quad 2+$ $1_{g}, \quad 1_{r}+1_{r}+1_{r}, \quad 1_{r}+1_{r}+1_{g}, \quad 1_{r}+1_{g}+1_{g}$ and $1_{g}+1_{g}+1_{g}+1_{g}$. This verifies Theorem 3.7(iii) for $j=1$ and $n=0$.

\section{ACKNOWLEDGEMENTS}

The author would like to thank the referee for his/her valuable comments, which helped to improve the manuscript.

\section{References}

Andrews, G. E. \& Berndt, B. C. (2009). Ramanujan's Lost Notebook Part II. Springer, New York.

Berndt, B. C. (1991). Ramanujan's Note books, Part III. Springer, New York.

Berndt, B. C. \& Rankin, R. A. (1995). Ramanujan: Letters and Commentary. American Mathematical Society.

Hirschhorn, M. D. \& Sellers J. A. (2005). Arithmetic relations for overpartitions. Journal of Combinatorial Mathematics and Combinatorial Computing, 53: 65-73.

Ramanujan, S. (1919). Some properties of $p(n)$, the number of partition of $n$. Proceedings of the Cambridge Philosophical Society, 19: 207-210

Ramanujan, S. (1998). The Lost Notebook and Other Unpublished Papers. Narosa, New Delhi.

Submitted : 07/07/2020

Revised : :25/10//2020

Accepted : 25/10/2020

DOI $\quad: 10.48129 / \mathrm{kjs} . v 48 \mathrm{i} 2.9907$ 